\title{
Elucidating the Mechanism of Vanillin Induced Mycobacterial Membrane Disruption: Implications of Lipid Alteration
}

\author{
Sharda Sharma ${ }^{1}$, Sandeep Hans ${ }^{1}$, Saif Hameed ${ }^{1 *}$ and Zeeshan Fatima ${ }^{{ }^{*}}$ \\ ${ }^{1}$ Amity Institute of Biotechnology, Amity University Haryana, Gurgaon (Manesar), 122413, India
}

Received: 21 March, 2017; Accepted: 28 April, 2017; Published: 10 May, 2017

*Corresponding authors: Zeeshan Fatima, Amity Institute of Biotechnology, Amity University Haryana, Gurgaon (Manesar)-122413, India. Tel: +91-124-2337015, Ext: 1116; E-mail: drzeeshanfatima@gmail.com

Saif Hameed, Amity Institute of Biotechnology, Amity University Haryana, Gurgaon (Manesar)-122413, India, Tel: +91-124-2337015, Ext: 1116; E-mail: saifhameed@yahoo.co.in

\begin{abstract}
Background: Novel strategies to overcome Multidrug Resistance (MDR) in Tuberculosis (TB) still remain a concern. Usage of natural compounds nowadays to surmount the ever increasing burden of MDR-TB has shown promising results.
\end{abstract}

Objectives: Previously, we have demonstrated the disruption of cell surface integrity as potential antimycobacterial mechanism of vanillin (Van), a natural food flavouring agent and preservative. This study aimed to gain deeper insights into the causal effects of membrane disruption.

Methods: Efflux and passive diffusion assay was performed with substrate EtBr. Changes in lipid profiles were studied by thin layer chromatography and lipase assay. PI uptake was done by fluorescence assay. Colony morphology and cell sedimentation were estimated microscopically and spectrophotometrically respectively.

Results: We reveal that membrane disruption leads to enhanced passive diffusion of drug independent of drug efflux activity. We further explored that Van caused alterations in lipid as demonstrated by impaired lipid profile and enhanced lipase activity. Commensurate with our findings, we also observed that Van leads to enhanced PI intake validating the hypothesis. Furthermore, we also uncover additional phenotypes related to membrane disruption. We observed that Van affects colony morphology and showed enhanced cell sedimentation rate depicting fluctuations in lipid composition.

Conclusion: Taken together, considering the emerging role of lipids in MDR phenotype, alterations in lipid as demonstrated through this study clearly warrants lipidomics based approach to discover novel targets for anti-TB therapeutics.

Keywords: Vanillin; Mycobacterium; Lipids; Passive Diffusion; Lipase; Colony Morphology

\section{Introduction}

In recent years, Tuberculosis (TB) has become one of the major dangerous chronic infectious disease causing high mortality and morbidity worldwide. According to World Health Organization (WHO) report there were estimated 1.8 million population deaths with TB in 2015 out of which 0.4 million deaths are only from HIV associated with TB [1]. The current anti-TB drug regimens which are used for the treatment of TB having different mechanisms of action after long term exposure suffers from development of multi drug resistance tuberculosis (MDR-TB) [2]. Mycobacterium tuberculosis (MTB), the causative agent of TB, possesses unique cell wall architecture which aides MTB to resist against several anti-TB drugs. Emergence of MDR along with adverse side effect on human health due to prolong anti-TB drugs usage has nowadays prompted to identify novel and effective drugs from natural origin to overcome this fatal disease $[3,4]$.

Vanillin (Van), a natural phenolic compound, is extracted from seed pods of Vanilla planifolia. It has pleasant smell and taste of vanilla which is commonly used as a flavoring agent and aroma in food, beverages and pharmaceuticals. Van is "generally regarded as safe" (GRAS) with an acceptable daily intake of 10 $\mathrm{mg} / \mathrm{kg}$ as agreed between FAO/WHO and EU [5]. Van already has therapeutic potential such as antimicrobial, anticarcinogenic, antioxidant and hypolipidemic properties [6-9]. Our group have deciphered the antifungal activity of Van against most prevalent human fungal pathogen Candida albicans (unpublished data). Moreover, we have previously reported that Van has promising antimycobacterial activity against Mycobacterium and affects membrane integrity, iron homeostasis and biofilm formation [10]. The present study, aimed to expand our understanding and add to the existing literature about the mechanisms involved in disruption of mycobacterial membrane integrity by Van, being the major target of currently used first line anti-TB drugs. The study explored that Van leads to enhanced passive diffusion of drugs independent of drug efflux pump activity and depicts alteration in lipid metabolism. This is also supported by the effect of Van on colony morphology and cell sedimentation rate. All these unreported mechanisms are significant for pathogenesis of MTB.

\section{Material and Methods}

All Media chemicals Middlebrook 7H9 broth, Middlebrook 7H10 agar, albumin/dextrose/catalase (ADC), 
oleic acid/albumin/dextrose/catalase (OADC) supplements was purchased from BD Biosciences (USA). Van was purchased from Sigma-Aldrich (St. Louis, MO, USA). TLC silica gel 60 F254 $(20 \times 20)$ was purchased from Merck.

\section{Methods}

\section{Strains and Growth Culture}

M. smegmatis $\mathrm{mc}^{2} 155$ was grown in Middlebrook $7 \mathrm{H} 9$ (BD Biosciences) broth supplemented with $0.05 \%$ Tween-80 (Sigma- Aldrich), 10\% ADC (BD Biosciences), and 0.2\% glycerol (Thermo Fischer Scientific) in 100-ml flasks and incubated at $37^{\circ} \mathrm{C}$ Cultures were subsequently grown on Middlebrook $7 \mathrm{H} 10$ (BD Biosciences) agar media supplemented with 10\% (v/v) OADC (BD Biosciences) for solid agar allowing growth for $48 \mathrm{~h}$ at $37^{\circ} \mathrm{C}$. Stock cultures of log-phase cells were maintained in $30 \%$ glycerol and stored at $-80^{\circ} \mathrm{C}$.

\section{Cell Viability Assay}

Cell viability assay was performed by using Trypan blue dye as described elsewhere [11]. Briefly, cells were grown at $37^{\circ} \mathrm{C}$ for $24 \mathrm{~h}$ in Middlebrook broth supplemented with $10 \%$ ADC in absence (control) and presence of Van. The cells and trypan blue $(0.4 \%)$ were diluted in the ratio $1: 1$ and kept at room temperature for $15 \mathrm{~min}$. $10 \mu \mathrm{l}$ bacterial suspension were transferred to hemocytometer, covered with cover-slip and both stained and unstained cells were counted under light microscope at $40 \mathrm{x}$ magnification.

\section{EtBr Efflux and Passive Diffusion}

The efflux of EtBr was determined by using protocol described previously [2]. Briefly, approximately $1 \times 10^{6}$ cells were grown until exponential phase in the absence (control) and in presence of Van at its sub-inhibitory concentration $(15 \mu \mathrm{g} / \mathrm{ml})$. Cells were pelleted, washed twice with Phosphate-Buffered Saline (PBS) (without glucose), and resuspended as a 2\% cell suspension. The cells were then de-energized with an efflux pump inhibitor 2, 4 DNP $(20 \mu \mathrm{g} / \mathrm{ml})$ in PBS (without glucose). The de-energized cells were pelleted, washed, and then resuspended as a $2 \%$ cell suspension $(\mathrm{w} / \mathrm{v})$ in PBS without glucose, to which EtBr was added at a final concentration of $4 \mu \mathrm{g} / \mathrm{ml}$ and incubated for $45 \mathrm{~min}$ at $30^{\circ} \mathrm{C}$. The equilibrated cells with EtBr were then washed and resuspended as a $2 \%$ cell suspension $(\mathrm{w} / \mathrm{v}$ ) in PBS (with glucose $0.4 \%$ ) for EtBr efflux and PBS (w/o glucose) for passive diffusion respectively. Samples with a volume of $2 \mathrm{ml}$ were withdrawn at different time interval and centrifuged at 10,000 rpm for $1 \mathrm{~min}$. The supernatant was collected, and absorption was measured at $285 \mathrm{~nm}$. Glucose-free samples as negative controls were included for both the EtBr efflux and passive diffusion experiments.

\section{Lipid Extraction and Thin Layer Chromatography (TLC)}

Untreated (control) and Van treated cells of $M$. smegmatis at exponential phase were used for lipid extraction by Folch method with slight modification [12]. Briefly, the cells were harvested at 10,000 rpm for $10 \mathrm{~min}$. Cells were homogenized in aqueous solution for $3 \mathrm{~min}$ and suspended in $\mathrm{CHCl}_{3}$ and $\mathrm{CH}_{3} \mathrm{OH}$ in ratio of (1:2). Cells were shaken well and centrifuged at $2000 \mathrm{rpm}$ at $4{ }^{\circ} \mathrm{C}$ for $15 \mathrm{~min}$. Supernatant was transferred to another glass vial and then remaining $\mathrm{CHCl}_{3}$ was added and filtered through Whatman No. 1 filter paper. The extract was then washed with $0.88 \% \mathrm{KCl}$ to remove the non-lipid contamination. The lower dense layer of chloroform containing lipid was taken by glass Pasteur pipette in $5 \mathrm{ml}$ glass vial with Teflon capping. The vials are stored at $20^{\circ} \mathrm{C}$ until further analysis. Extracted lipids were then resolved by TLC using aluminium-backed silica gel plates (silica gel 60 F254; Merck). The lipid extract obtained from control and Van was loaded on TLC plate at a distance of $2 \mathrm{~cm}$ up from the plate end. Chloroform-methanol-water $(65: 25: 4 ; \mathrm{v} / \mathrm{v} / \mathrm{v})$ was used for developing the plates. Developed chromatogram was dried at room temperature for $2 \mathrm{~min}$ and then exposed to iodine fumes generated by iodine crystal balls placed in glass chamber to visualize the lipids.

\section{Lipase Assay}

M. smegmatis cells were grown in Middlebrook 7H9 broth in the absence (control) and presence of Van $(15 \mu \mathrm{g} / \mathrm{ml})$. Whole cell protein was extracted [13] and protein concentration was determined by Lowry method as previously described [14]. Lipase activity was performed by measuring the amount of p-nitrophenol ( $\mathrm{p}$-NP) released from $\mathrm{p}$-NP ester substrate with varying lengths of fatty acids. The total lipase activity was assayed as using protein extract of M. smegmatis. The standard lipase activity assays were performed in $100 \mu \mathrm{l}$ reaction system consisting of a final concentration of $0.5 \mathrm{mM}$ p-NP esters substrate and the buffer ( $\mathrm{pH} \mathrm{8.0)}$ of $80 \mathrm{mM} \mathrm{H}_{3} \mathrm{BO}_{3}, 80 \mathrm{mM} \mathrm{H}_{3} \mathrm{PO}_{4}$, $300 \mathrm{mM} \mathrm{NaCl}, 0.3 \%$ Triton X-100 and $20 \%$ glycerol. The reaction mixture of purified protein was incubated at $37^{\circ} \mathrm{C}$ for $40 \mathrm{~min}$ and the release of p-nitrophenol was determined by measuring spectrophotometrically at $405 \mathrm{~nm}$ [15]. Cell extract free sample as negative control was included in the experiment.

\section{Acid -Fast Staining}

Briefly, cells were grown at $37^{\circ} \mathrm{C}$ for $24 \mathrm{~h}$ in Middlebrook broth supplemented with $10 \% \mathrm{ADC}$ in the absence (control) and presence of Van $(125 \mu \mathrm{g} / \mathrm{ml})$. Prepare bacterial smear on clean and grease free slide, using sterile technique. Allow smear to air dry and then heat fix. Cover the smear with carbol fuchsin stain and heat for another 8-10 min. Then slide is then flooded with acid decolourizer to remove excess carbol fuchsin. Then cells were stained with methylene blue for $30 \mathrm{sec}$. Finally wash off the stain with clean water and observed under the microscope at 100x [16].

\section{Propidium Iodide Uptake Assay}

Propidium Iodide (PI) uptake assay was performed as described elsewhere with modification [17]. Briefly, cells were grown at $37^{\circ} \mathrm{C}$ for $24 \mathrm{~h}$ in Middlebrook broth supplemented with $10 \%$ ADC with or without Van. The cells were harvested by centrifugation and suspended in PBS to 0.5 Optical Density (O.D.) units at $600 \mathrm{~nm} .50 \mu \mathrm{g}$ PI was added in $1 \mathrm{ml}$ bacterial suspension were placed in each tube and kept at room temperature for $15 \mathrm{~min}$ in the dark. $10 \mu$ l bacterial suspension were transferred to a glass slide, covered with cover-slip and examined under fluorescence 
microscope at 40x.

\section{Colony Morphology}

Colony morphology was determined as by a protocol as described elsewhere [18]. Briefly, cells were plated in the presence and absence of Van on Middlebrook agar plates supplemented with $10 \%$ OADC and incubated at $37^{\circ} \mathrm{C}$ for 2 to 4 days. Post incubation, images of the individual colonies were taken at $10 \mathrm{x}$ magnification.

\section{Cell Sedimentation Assay}

Cell sedimentation assay was performed as described elsewhere [18]. Cultures at $\mathrm{OD}_{600} \sim 1.0-1.4$ of the control and cells treated with sub-inhibitory concentration $15 \mu \mathrm{g} / \mathrm{ml}$ in Middlebrook media supplemented with ADC were adjusted in triplicate to $\mathrm{OD}_{590} \sim 1.0$ and kept unshaken at $37^{\circ} \mathrm{C}$. At 3 and 22 hours, the upper $1 \mathrm{ml}$ was removed for $\mathrm{OD}_{590}$ measurements.

\section{Results}

\section{Van is Bacteriostatic in Nature}

In order to assess the effect of Van on mycobacterial growth, firstly we confirmed whether Van was bacteriostatic or cidal in nature. Our results demonstrated that when cells grown at MIC concentration of Van and re-inoculated in fresh media, cells were efficiently revived irrespective of the presence of Van confirming its bacteriostatic nature (Figure 1a). Based on the static nature, we next tested the effect of Van on mycobacterial cell viability. We explored that cell viability was 53\% preserved in presence of Van in comparison to the untreated cells (Figure 1b).

(a)

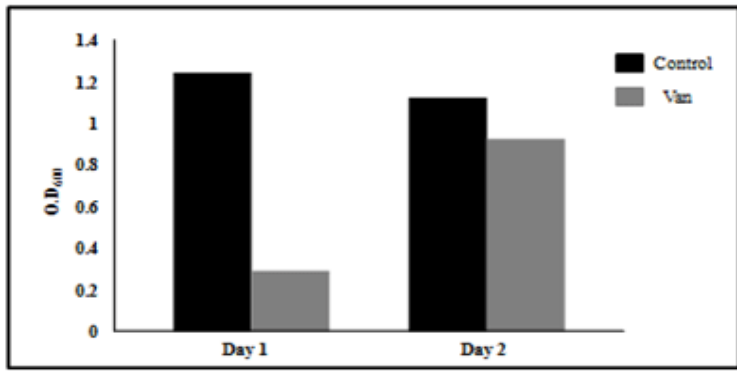

(b)

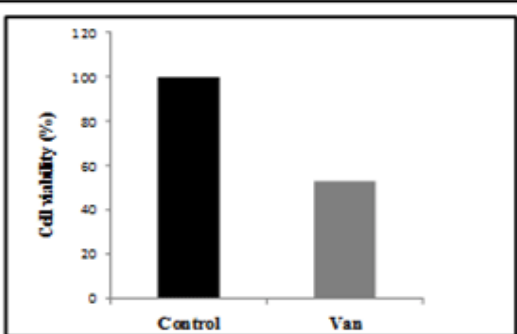

Figure 1: Effect of Van on cell viability of $M$. smegmatis

(a) Cellular growth (O.D 600) is depicted on y-axis in the absence (control) and presence of Van $(125 \mu \mathrm{g} / \mathrm{ml})$ on $\mathrm{x}$-axis on day 1 and after reinoculation on day 2 respectively.

(b) \% viability of $M$. smegmatis cells is depicted on y-axis in absence (control) and presence of Van $(125 \mu \mathrm{g} / \mathrm{ml})$ on x-axis.

\section{Van Does not Affect Efflux Pump Activity Instead Showed Enhanced Passive Diffusion}

Over expression of drug efflux pumps is the major mechanism of drug resistance encountered in MTB [19-21]. To rule out the possibility of any involvement of abrogated efflux of drug towards antimycobacterial activity of Van, we performed EtBr efflux assay in the presence of Van. We observed that there was no significant difference in the activity of efflux pump in the presence of Van (Figure 2a). Furthermore, we performed passive diffusion assay to investigate whether Van affects passive diffusion of drug instead of efflux pump activity. Interestingly, our result depicted increased extracellular concentration of EtBr in de-energized cells indicating enhanced passive diffusion in the presence of Van in contrast to control (Figure 2b). (a)

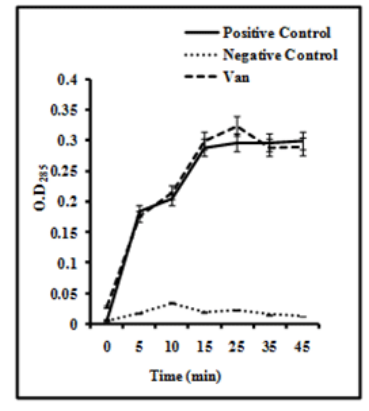

(b)

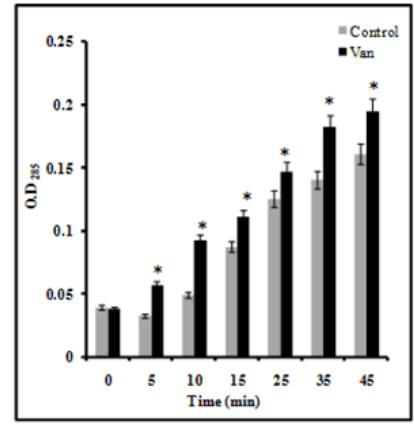

Figure 2: Effect of Van on drug efflux assay and passive diffusion of M. smegmatis.

(a) Drug efflux assay shown by extracellular concentration of EtBr in the absence (control) and presence of Van $(15 \mu \mathrm{g} / \mathrm{ml})$. Mean of O.D $285 \pm$ SD of three independent sets of experiments are depicted on $y$-axis with respect to time (minutes) on $\mathrm{x}$-axis.

(b) Passive diffusion of de-energized $M$. smegmatis cells displayed by extracellular concentration of $\mathrm{EtBr}$ in absence (control) and presence of Van $(15 \mu \mathrm{g} / \mathrm{ml})$. Mean of O.D $285 \pm$ SD of three independent sets of experiments are depicted on y-axis with respect to time (minutes) on $\mathrm{x}$-axis. * depicts $P$ value $<0.05$.

\section{Van Exerts Lipid Alterations}

Next, we confirm whether the membrane disrupting effect of Van is due to any lipid alterations or not. For this total cellular lipid was isolated in presence of Van and Thin Layer Chromatography (TLC) was performed. The chromatogram displayed that there was significant alterations in the lipid profile in presence of Van (Figure 3a). To substantiate this alteration, we further estimated the lipase activity in response to Van. To our expectation, we observed lipase activity of Mycobacterium was considerably enhanced in the presence of Van Figure $3 \mathrm{~b}$. We further tested the acid-fast staining in presence of Van and intriguingly we observed that the staining was diminished in presence of Van (Figure 3c). 
(a)

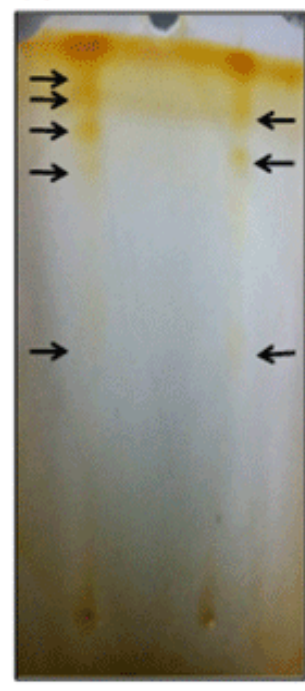

Control Van (b)

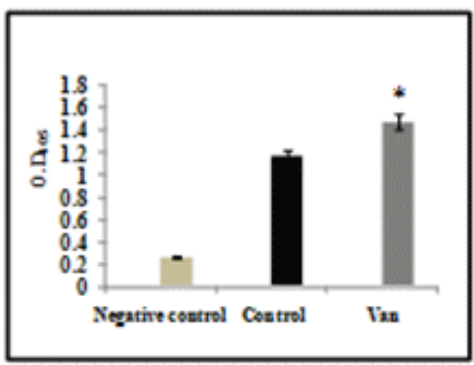

(c)

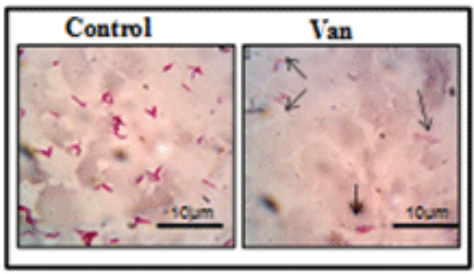

Figure 3: Effect of Van induced lipid alteration in M. smegmatis. (a) Thin layer chromatogram showing altered total lipid profile in the presence of Van.

(b) Lipase activity quantified by using lipase assay and depicted as bar graph in the absence (control) and presence of Van. Mean of O.D $405 \pm$

SD of three independent sets of experiments are depicted on Y-axis and * depicts $P$ value $<0.05$.

(c) Acid fast staining showing bright pink stain in the control cells contrary to light pink colour depicting less staining in presence of Van. The images were taken at $100 x$.

\section{Van Leads to Enhanced PI Uptake Depicting Non-Intact Membrane}

Additionally, Van causes membrane disruption and alteration in lipid composition was further apparent from PI mediated fluorescence assay. PI is a dye known which is known to bind nucleic acids only when membrane is not intact or broken due to any stress. Our result depicts that in presence of Van we observed red colour fluorescence in contrast to untreated cells (control) where we observed no fluorescence implying membrane lesions, decreasing membrane integrity and increasing membrane permeability (Figure 4).

\section{Van Leads to Altered Colony Morphology and Enhanced Cell Sedimentation Rate}

Finally we assessed whether the alteration in lipid metabolism due to Van is responsible for any alteration in the surface properties of Mycobacterium which largely comprises lipid moieties. As expected, we observed colony with rough and undefined borders in the presence of Van in comparison to control colony which depicts smooth and waxy borders (Figure 5a). We also estimated the cell sedimentation rate which could be related with any alterations in cell surface properties. Our observation suggests that cell sedimentation rate is considerably enhanced in presence of Van (Figure 5b).

(a)

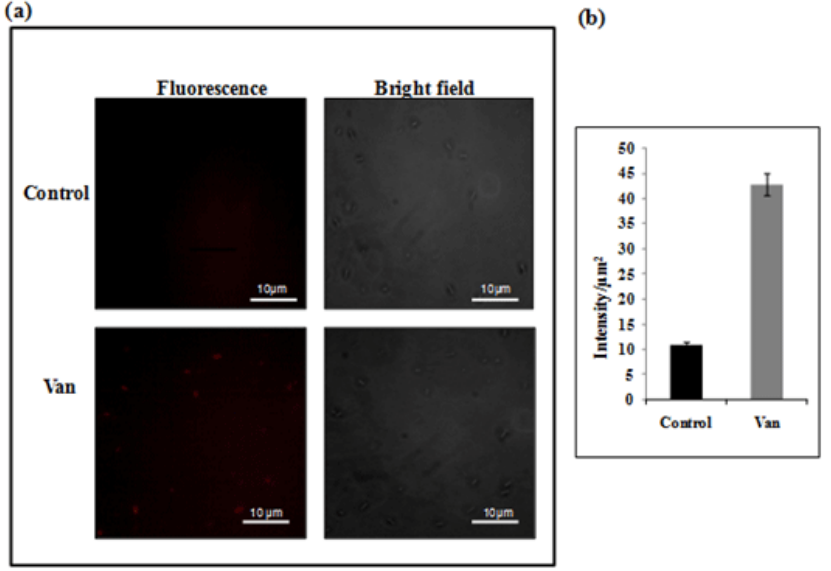

Figure 4: Effect of Van on PI uptake assay of M. smegmatis.

(a) Fluorescence microscopy observation of PI stained cells in the absence (control) and presence of Van. Cells with membrane damage are stained with PI (red signals) in contrast to intact membrane of control cells. The images were taken at $40 \mathrm{x}$.

(b) Fluorescence intensity of PI stained cells depicted as bar graph in the absence (control) and presence of Van.

(a)

(b)
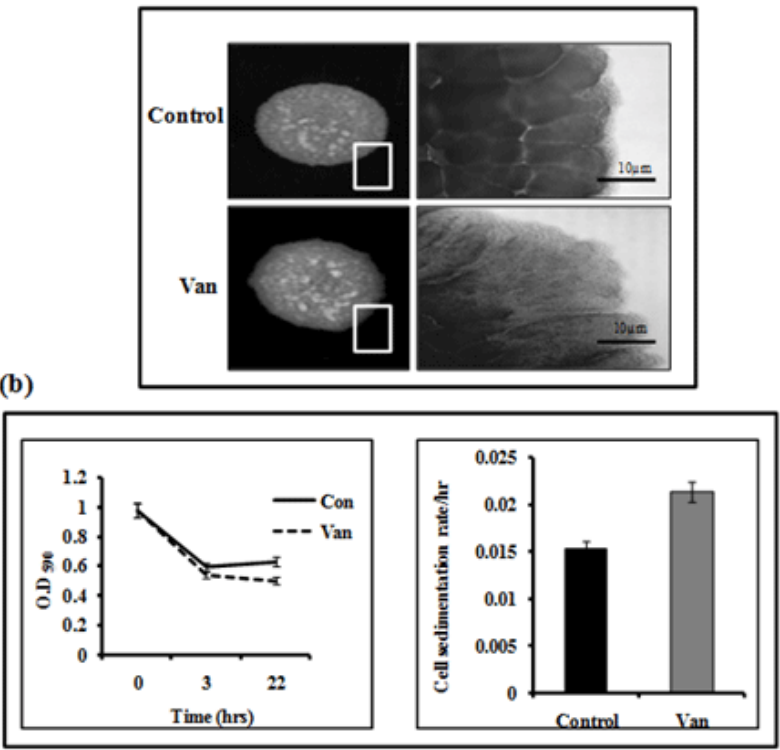

Figure 5: Effect of Van on cell surface properties of $\boldsymbol{M}$. smegmatis. (a) Altered colony morphology in the presence of Van (10x magnification). Colony morphology of control and Van treated cells were observed on 7H10-based medium. Corresponding area of colony borders (left panel) were focused to show the borders (right panel).

(b) Relative sedimentation of $M$. smegmatis cells in the presence of Van. Left panel shows O.D 590 of untreated (control) and Van treated cells depicted on Y-axis with respect to time noted at 3 and $22 \mathrm{~h}$ on $\mathrm{X}$-axis. Right panel depicts sedimentation rates per hour on Y-axis of Van treated cells with respect to control on X-axis, calculated by estimating the difference in O.D 590 from 0 till 22 h per unit time interval. Mean of three independent sets of experiments \pm SD (shown by error bars) are depicted. 


\section{Discussion}

Van is known to act as a potent antimicrobial against both fungi and bacteria. For instance, antifungal activity of Van against Cryptococcus neoformans has been reported earlier [22]. The antibacterial effects of Van mixtures with Cinnamon and clove essential oil in controlling Listeria monocytogenes and Escherichia coli 015:h7 in milk is already reported [23]. Similarly, another study has revealed the mode of antimicrobial action of Van against Escherichia coli, Lactobacillus plantarum and Listeria innocua. Insights into the mechanism revealed that Van alters membrane integrity, decrease in respiration and loss of $\mathrm{K}^{+}$and $\mathrm{H}^{+}$ion gradients [24]. In search for the discovery of novel drugs (preferably from natural sources) with novel targets, previously we have demonstrated the antimycobacterial potential of Van, a natural food flavouring agent albeit bacteriostatic as observed from this study [10]. The study revealed that Van affects membrane integrity and virulence traits of mycobacteria.

Mycobacterium possesses unique cell wall characteristics such as presence of peptidoglycan, arabinogalactan (AG) and mycolic acid. The peptidoglycan is covalently attached to arabinogalactan layer, which is covered by mycolic acid. Presence of large amount of mycolic acid on the cell wall provides the effective barrier against the anti-TB drug. The cell membrane of MTB contains broad diversity of lipid molecules that are responsible for pathogenesis [25-27]. The disruption of membrane integrity in presence of Van as reported previously and role of lipids in development of MDR prompted us further to elucidate its mechanism and involvement of lipid associated changes in the present study $[10,28]$.

The efflux pumps are the transporter proteins which are present on the MTB membrane and involved in the movement of toxic substrates from cell to external environment. However in due course of time the over expressions of the efflux pumps have become the major mechanism of developing MDR in MTB [29] Therefore we firstly investigated whether membrane disruption of Van observed in previous study is due to any changes in the activity of efflux pump or not [10]. However, our results could safely conclude that the antimycobacterial mechanism of Van is independent of efflux pump inhibition (Figure 2a). Contrary, we could observe enhanced passive diffusion in presence of Van supporting the notion of membrane disruption (Figure 2b).

For deeper insights and to asses any alterations in lipid composition being the causal reason for enhanced passive diffusion across membrane, we studied the lipid profile in presence of Van by TLC. The chromatogram showed that total cellular lipids in control displayed various lipid classes at their respective positions in contrast to the Van treated lipids which were either absent or not detected at similar positions (Figure $3 a$ ). However, further work is still needed to precisely pin point the particular class of lipids getting severely affected. That Van leads to changes in lipid metabolism became further apparent from the fact that lipase activity was considerably enhanced in presence of Van (Figure 3b). Lipases are enzymes that occur universally in plant microbial and animal kingdoms. These enzymes play very vital role in fat digestion, lipoprotein metabolism, and mobilization of fat stored in lipid inclusion bodies, endosperms and adipocytes. Lipases catalyze the hydrolysis of ester bond of triacylglycerol to fatty acid and glycerol which are water soluble [30]. Lipase enzymes because it is involved in lipid metabolism are responsible for virulence factor of Mycobacterium [15]. Our hypothesis was further strengthened by the fact that acid-fast staining was considerably diminished in presence of Van (Figure 3c). Based on these observations one could hypothesize that Van causes alterations in lipid which could lead to changes in cell membrane of Mycobacterium. To further verify that Van causes alteration in lipid leading to membrane disruption, we performed a fluorescence assay of PI uptake. PI is a membrane impermeant dye that is excluded from viable cells. It binds to double stranded nucleic acids (DNA) by intercalating between the base pairs. If any disruption occurs on the cell membrane then it will enter inside the cell and give red colour fluorescence [31]. To our expectation, we could observe red fluorescence only in Van treated cells confirming non-intact membrane in comparison to untreated cells which showed no fluorescence depicting intact membrane (Figure 4). We therefore confirmed that Van affects mycobacterial cells by damaging the cell membrane and causing membrane lesions.

Taking clue from above observations and to phenotypically ascertain that Van leads to alterations in cell surface morphology constituted by various classes of lipid, we studied the colony morphology of Mycobacterium in presence of Van. Colony morphology is known to show the morphological characteristics of a bacterial colony such as size, texture, shape, elevation and effect on growth medium. Mycobacterium cell wall consists of lipid-rich, hydrophobic structure which is responsible for distinctive colony morphology, acid-fast staining and provide MDR properties. Mycobacterium posses hydrophobic lipid which gives the smooth surface that contribute virulence factor of Mycobacterium. From the previous studies it is known that cell surface hydrophobicity affect the colony morphology of Mycobacterium [18]. Alteration in membrane lipid may therefore interfere with colony morphology of Mycobacterium [32]. Additionally, colony morphology is also directly correlated with biofilm formation. The extracellular matrix associated with biofilms is notably rich in lipids. Free mycolic acids and Glycopeptidolipid (GPL) are associated with the formation of biofilms in Mycobacterium. The defective mycolic acid biosynthesis and GPL have impaired biofilm formation and morphology of the Mycobacterium [33]. The smooth colony with defined border describes surface properties of Mycobacterium which is involve in pathogenesis [34]. Interestingly, in presence of Van, we could observe colonies which have undefined and rough borders (Figure 5a). To further substantiate our findings, cell sedimentation rate in presence of Van was monitored that could be attributed to changes in cell surface hydrophobicity [18, 34]. We estimated that cell sedimentation rate was enhanced in presence of Van possibly due to the fact that cell envelope of mycobacteria have unique lipid classes which are known to govern pathogenicity and that Van could have led to certain alterations in those lipid classes (Figure $5 \mathrm{~b}$ ). 


\section{Conclusion}

Together, the data generated from this study has led us a step further to propagate the prospective of Van being considered as potential candidate for novel drug discovery (Figure 6) Moreover, with the advent of mass spectrometry based lipidomics approach, alterations in lipid metabolism in response to Van from this study warrants further attention to search for novel anti-TB drug targets.

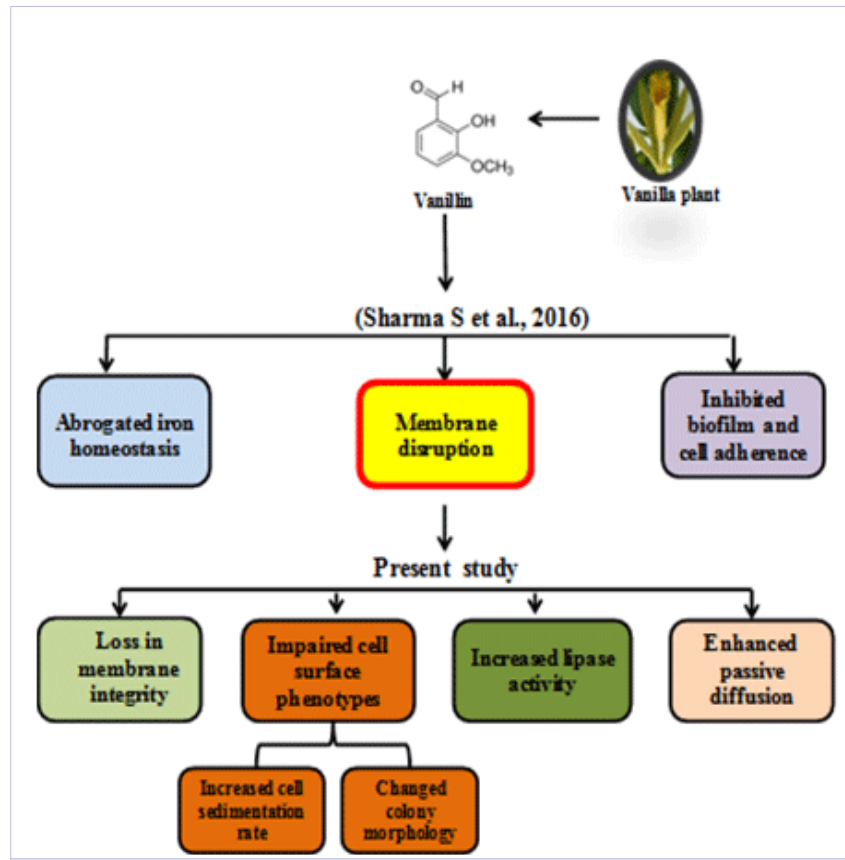

Figure 6: Mechanisms of Van induced mycobacterial membrane disruption.

\section{Acknowledgment}

We are grateful to Prof. Sarman Singh for providing $M$. smegmatis $\mathrm{mc}^{2} 155$ reference strain as generous gift.

\section{Acknowledgment}

1. WHO. World Health Organization report. 2016.

2. Pal R, Hameed S, Fatima Z. Iron Deprivation Affects Drug Susceptibilities of Mycobacteria Targeting Membrane Integrity. ] Pathog. 2015; 2015:938523.

3. Samad A, Sultana Y, Akhter MS, Agil M. Treatment of Tuberculosis: use of active pharmaceuticals. Recent Pat. Antiinfect Drug Discov. 2008; 3(1): 34-44.

4. Sharma S, Hameed S, Fatima Z. Natural compounds for overcoming Multidrug Resistance in Mycobacteria. Recent Pat Biotechnol. 2016;10(2):167-174.

5. Inchem (International Programme on Chemical Safety-INCHEM). Summary of Evaluations Performed by the Joint FAO/WHO Expert Committee on Food Additives. Hamilton, Ontario, CN: Canadian Centre for Occupational Health and Safety. 2004.

6. Ngarmsak M, Delaquis P, Toivonen P, Ngarmsak T, Ooraikul B, Mazza G. Antimicrobial activity of vanillin against spoilage micro-organism in stored fresh cut mangoes. J Food Prot. 2006;69(7):1724-1727.
7. Imanishi H, Sasaki YF, Matsumoto K, Watanabe M, Ohta T, Shirasu Y, et al. Suppression of 6-TG-resistant mutations in V79 cells and recessive spot formations in mice by vanillin. Mutat Res. 1990;243(2):151-158.

8. Burri J, Gra M, Lambelet P, Loliger J. Vanillin: more than a flavouring agent- a potent antioxidant. J Sci Food Agric. 1989;48(1): 49-56.

9. Mokshagundam LRV, Mokshagundam SL. Triglyceride reducing agent. 2003; US Patent 6,599,522.

10. Sharma S, Pal R, Hameed S, Fatima Z. Antimycobacterial mechanism of vanillin involves disruption of cell-surface integrity, virulence attributes, and iron homeostasis. Int J Mycobacteriol. 2016;5(4):460468. doi: 10.1016/j.ijmyco.2016.06.010

11. Tran SL, Puhar A, Ngo-Camus M, Ramarao N. Trypan blue dye enters viable cells incubated with pore- forming toxin HIyII of Bacillus cereus. PLoS One. 2011;6(9):e22876. doi: 10.1371/journal.pone.0022876

12. Pal R, Hameed S, Kumar P, Singh S, Fatima Z. Comparative Lipidome Profile of Sensitive and Resistant Mycobacterium tuberculosis Strain. Int. J Curr Microbiol App Sci. 2015;2015(1):189-197.

13. Lanigan MD, Vaughan JA, Shiell BJ, Beddome GJ, Michalski WP. Mycobacterial proteome extraction: comparison of disruption methods. Proteomics. 2004;4(4): 1094-1100.

14. Lowry OH, Rosebrough NJ, Farr AL. Protein measurement with the Folin phenol reagent. J Biol Chem. 1951;193(1):265-275.

15. Cao J, Dang G, Li H, Li T, Yue Z, Li N, et al. Identification and Characterization of Lipase Activity and Immunogenicity of LipL from Mycobacterium tuberculosis. PLoS One. 2015;10(9):e0138151. doi: 10.1371/journal.pone.0138151

16. Treuer R, Haydel SE. Acid-Fast Staining and Petroff Hausser Chamber Counting of Mycobacterial Cells in Liquid Suspension. Curr Protoc Microbiol. 2011; Chapter 10: Unit 10A.6. doi: 10.1002/9780471729259.mc10a06s20

17. Shi L, Gunther S, Hubschamann T, Wick LY, Harms H, Muller S. Limits of Propidium Iodide as a Cell Viability Indicator for Environmental Bacteria. Cytometry A. 2007;71(8): 592-598.

18. Pal R, Hameed S, Sharma S, Fatima Z. Influence of iron deprivation on virulence traits of mycobacteria. Braz J Infect Dis. 2016;20(6):585591. doi: 10.1016/j.bjid.2016.08.010.

19. Pal R, Fatima Z, Hameed S. Efflux pumps in drug resistance of Mycobacterium tuberculosis. A panoramic view. Int J Curr Microbiol Appl Sci. 2014; 3(8): 528-546.

20. Jin J, Zhang JY, Guo N, Sheng H, Li L, Liang JC, et al. Farnesol a potential efflux pump inhibitor in Mycobacterium smegmatis. Molecules. 2010;15(11):7750-7762. doi: 10.3390/molecules15117750

21. Lechner D, Gibbons S, Bucar F. Plant phenolic compounds as ethidium bromide efflux inhibitors in Mycobacterium smegmatis. J Antimicrob Chemother. 2008;62(2):345-348. doi: 10.1093/jac/dkn178

22. Boonchird C, Flegel TW. In vitro antifungal activity of eugenol and vanillin against Candida albicans and Cryptococcus neoformans. Can J Microbiol. 1982;28(11):1235-1241.

23. Roda RMC, Rodriguez AT, Franco MTV, Iniesta FM. Antimicrobial Activity of Vanillin and Mixture with Cinnamon and Clove Essential Oil in Controlling Listeria Monocytogenes and Escherichia coli 0157:H7 in Milk. Food Bioprocess Technol. 2012;5(6):2120-2131. doi: 10.1007/ s11947-010-0484-4

24. Fitzgerald DJ, Stratford M, Gasson MJ, Ueckert J, Bos A, Narbad A. 
Mode of antimicrobial action of vanillin against Escherichia coli, Lactobacillus plantarum and Listeria innocua. J Appl Microbiol. 2004; 97(1):104-113.

25. Forrellad MA, Klepp LI, Gioffré A, Sabio y García J, Morbidoni HR, de la Paz Santangelo M, et al. Virulence factors of the Mycobacterium tuberculosis complex. Virulence. 2013;4(1):3-66. doi: 10.4161/ viru.22329

26. Alderwick, LJ, Birch HL, Mishra AK, Eggeling L, Besra GS. Structure, function and biosynthesis of the Mycobacterium tuberculosis cell wall: Arabinogalactan and lipoarabinomannan assembly with a view to discovering new drug targets. Biochem Soc Trans. 2007;35(Pt 5):1325-1328.

27. Brennan, PJ. Structure, function and biogenesis of the cell wall of Mycobacterium tuberculosis. Tuberculosis (Edinb). 2003;83(1-3):9197.

28. Sun T, Qin B, Gao M, Yin Y, Wang C, Zang S, et al. Effects of epigallocatechin gallate on the cell-wall structure of Mycobacterium smegmatis mc2155. Nat Prod Res. 2014;29(22):2122-2124. doi:10.1 $080 / 14786419.2014 .989391$
29. Groblacher B, Kunert O, Bucar F. Compounds of Alpinia katsumadai as efflux inhibitors in Mycobacterium smegmatis. Bioorg Med Chem. 2012;20(8):2701-2706. doi: 10.1016/j.bmc.2012.02.039

30. Patil KJ, Chopda MZ, Mahajan RT. Lipase biodiversity. Indian J Sci Technol. 2011; 4(8):971-982.

31. Soejima T, Iida K, Qin T, Taniai H, Yoshida S. Discrimination of live, antituberculosis agent- injured and dead Mycobacterium tuberculosis using flow cytometry. FEMS Microbiol Lett. 2009;294(1):74-81. doi: 10.1111/j.1574-6968.2009.01549.x

32. Pacheco SA, Hsu FF, Powers KM, Purdy GE. MmpL11 protein transports mycolic acid-containing lipids to the mycobacterial cell wall and contributes to biofilm formation in Mycobacterium smegmatis. J Biol Chem. 2013;288(33):24213-24222. doi: 10.1074/jbc.M113.473371

33. Chen JM, German GJ, Alexander DC, Ren H, Tan T, Liu J. Roles of Lsr2 in Colony Morphology and Biofilm Formation of Mycobacterium smegmatis. J Bacteriol. 2006; 188(2): 633-641.

34. Jamet S, Slama N, Domingues J, Laval F, Texier P, Eynard N, et al. The Non-Essential Mycolic Acid Biosynthesis Genes hadA and hadC Contribute to the Physiology and Fitness of Mycobacterium smegmatis. PLoS One. 2015;10(12):e0145883. 